\title{
Comment on Madelaine et al.: Results and complications of single-stage total knee arthroplasty and high tibial osteotomy
}

\author{
Abhay Elhence • Divesh Jalan • Devendra Singh Rathore • \\ Maley Deepak Kumar • Hemant Chahar
}

Received: 30 October 2014 / Accepted: 27 November 2014 / Published online: 24 December 2014

(C) SICOT aisbl 2014

Dear Editor,

We read the article by Madelaine et al. [1] entitled "Results and complications of single-stage total knee arthroplasty and high tibial osteotomy" with keen interest. We appreciate the authors' work; however, we would like to point out certain issues, which we believe will contribute to the effectiveness of the study.

1. The authors mention measurement of the femoral mechanical axis (mFA), tibial mechanical axis (mTA) and femorotibial mechanical axis (mFTA) on weight-bearing long-leg films to determine the magnitude and location of the extra-articular deformity. However, it is not clear from the study as to how the authors have arrived at these angles and how they have concluded that the deformity measured is an extra-articular deformity.

2. The authors recommend grafting the osteotomy defect with autograft bone from the bony cuts. It is, however, not clear from the article whether the same technique had been used in their study.

3. We would be curious to know whether the autograft introduced into the valgising osteotomy had been placed before or after the introduction of the tibial stem. It is our understanding that both of the above clinical scenarios would lead to damage to the autograft, as is evident from all the postoperative $\mathrm{X}$-rays published in the article which show a valgus tibial stem abutting on to the lateral tibial cortex, which indicates either inadequate correction or loss of correction of the deformity.

A. Elhence $\cdot$ D. Jalan · D. S. Rathore $(\bowtie) \cdot$ M. D. Kumar · H. Chahar

Department of Orthopaedics, All India Institute of Medical Sciences, Basni Industrial Area Phase-2, Jodhpur 342005, Rajasthan, India e-mail: devendra01.86@gmail.com
4. It is our opinion that single-stage correction for a severe extra-articular deformity entails a significantly higher risk of nonunion, infection and periprosthetic fracture with increased duration of immobilisation, as has also been reported by the authors in this study. It is only prudent to consider a two-stage surgery, thereby minimising the risk of complications and justifying the need for total knee replacement (TKR) which is to provide early, painless mobilisation in a well-balanced stable knee.

5. It is our submission that conversion to a TKR after high tibial osteotomy (HTO) is a difficult surgery with its antecedent problems, viz. offset stem and alteration of posterior slope: does performing two difficult operations in a single stage compromise the long-term survivorship of a TKR?

6. The authors have concluded that the combination of TKR and HTO is a valid option for treating arthrosis with large extra-articular frontal plane deformity. However, recent studies $[2,3]$ have concluded that good pre-operative planning, intra-articular bone resection and soft tissue balancing (both in flexion and extension) would be able to correct the majority of the extra-articular deformities.

\section{References}

1. Madelaine A, Villa V, Yela C, Lording T, Lustig S, Servien E, Neyret P (2014) Results and complications of single-stage total knee arthroplasty and high tibial osteotomy. Int Orthop 38(10):2091-2098

2. Rajgopal A, Vasdev A, Dahiya V, Tyagi VC, Gupta H (2013) Total knee arthroplasty in extra articular deformities: a series of 36 knees. Indian J Orthop 47(1):35-39

3. Koenig JH, Maheshwari AV, Ranawat AS, Ranawat CS (2009) Extraarticular deformity is always correctable intra-articularly: in the affirmative. Orthopedics Sep;32(9) 